\title{
Alternativas de diseño para mejorar el desempeño ambiental de plazas urbanas de Mendoza (Argentina). Evaluación mediante simulación con ENVI-met 3.1
}

\author{
Design alternatives for environmental improvements in urban squares \\ of Mendoza - Argentina. Evaluation by means of ENVI-met 3.1
}

$\underline{\text { S. Stocco }}^{(*)}$, A. Cantón ${ }^{(*)}$, E. Correa ${ }^{(*)}$

RESUMEN

Este trabajo tiene como objetivo determinar el potencial de las estructuras que componen las plazas verdes urbanas: arbolado de gran porte, áreas de césped y superficies selladas de forma aislada. Con este fin se evaluó el comportamiento ambiental de una plaza compuesta al 100\% por cada estructura. Para la caracterización térmica y radiativa se realizaron simulaciones con ENVI-met 3.1. Los resultados muestran que la cobertura de la plaza con arbolado de gran porte al 100\% mejora las condiciones de habitabilidad durante el día y presenta las menores temperaturas durante la noche, con diferencias de hasta $3.50^{\circ} \mathrm{C}$ respecto de la peor configuración (superficie sellada 100\%). Si bien es imprescindible que las plazas contengan estructuras selladas para dar respuesta a sus requerimientos funcionales, desde el punto de vista ambiental, las plazas debieran priorizar en sus diseños grupos de árboles de gran porte y disminuir la presencia de estructuras selladas.

Palabras clave: Plazas verdes, Simulación numérica, ENVI-met 3.1, Árboles de gran porte.

\section{ABSTRACT}

This investigation aims to determine the potential of each of the structures that make up the urban squares: forest large size, grass areas and sealed surfaces in isolated way. For this purpose, the environmental performance of three urban squares constituted at $100 \%$ by each structure was evaluated. Numerical simulations with ENVI-met 3.1 were performed for its thermal and radiative characterization. The results show that the coverage of the square with forest large size to $100 \%$ improves the conditions of habitability during the day and presents the lower temperatures during the night, with differences of up to $3.5^{\circ} \mathrm{C}$ in relation to the worst configuration (sealed surface $100 \%$ ). It is essential that the urban squares contain sealed structures to meet their functional requirements. Nevertheless, based in this study, urban squares should prioritize forest large size groups in their designs and decrease the presence of sealed structures to get better performance from the environmental standpoint.

Keywords: Green square, Numerical simulation, ENVI-met 3.1, Forest large size.

(*) Consejo Nacional de Investigaciones Científicas y Técnicas (CONICET) Argentina Persona de contacto/Corresponding author: sstocco@mendoza-conicet.gob.ar (S. Stocco) ORCID: http://orcid.org/oooo-0002-8153-5386 (S. Stocco); http://orcid.org/oooo-0oo2-8714-9697 (A. Cantón); http://orcid.org/oooo-0003-1690-076X (E. Correa)

Cómo citar este artículo/Citation: S. Stocco, A. Cantón; E. Correa (2018). Alternativas de diseño para mejorar el desempeño ambiental de plazas urbanas de Mendoza (Argentina). Evaluación mediante simulación con ENVI-met 3.1. Informes de la Construcción, 70(550): e253. https://doi.org/10.3989/ic.16.154

Copyright: (C) 2018 CSIC. Este es un artículo de acceso abierto distribuido bajo los términos de la licencia de uso y distribución Creative Commons Reconocimiento 4.0 Internacional (CC BY 4.0). 


\section{INTRODUCCIÓN}

El enverdecimiento urbano es una de las principales estrategias de mitigación de la artificialización del medio natural que ayuda a mejor las condiciones ambientales y de habitabilidad del espacio urbano-edilicio (1) (2) (3). En las últimas décadas se ha incrementado la conciencia y el conocimiento sobre la importancia que revisten los espacios verdes, debido a los beneficios que producen (4) (5) (6) (7). Hacer énfasis en el desarrollo de propuestas de diseño que contemplen tanto el aspecto ambiental como el paisajístico de estos espacios permitirá potenciar sus beneficios y mejorar la calidad de vida de los habitantes.

De modo particular la ciudad de Mendoza en Argentina, a diferencia de otras ciudades de clima árido, presenta intensa vegetación. Desde sus principios la ciudad estuvo compuesta por calles y veredas anchas de 16,20 y $30 \mathrm{~m}$, fuertemente forestadas y espacios verdes conformados por: Bulevares, Paseos, Plazoletas, Plazas y Parques (Ver figura 1). Entre ellos "las plazas" son relevantes por ocupar $212549 \mathrm{~m}^{2}$ de la trama urbana. La ciudad contiene 14 plazas que representan el 30\% de sus espacios verdes, ofreciendo gran potencial a nivel urbano, para mitigar el efecto de isla de calor a escala macro y mejorar la habitabilidad térmica de la trama a escala micro (8) (9) (10).

En trabajos previos (5) (11) se identificaron patrones de diseño relevantes para las plazas de Mendoza, donde predomina el esquema simétrico de organización interna con concentración del área sellada en el centro de la plaza. Respecto a su configuración espacial se destaca la presencia de tres estructuras, con características diferentes que se repiten en todos los casos de estudio, se las denomina: Áreas de arbolado de gran porte, de césped y superficies selladas. Se define como arbolado de gran porte al área donde predomina vegetación de 15 a 30 metros de altura cuyo solapamiento de copas genera áreas de sombra y huecos de tamaños variables. Áreas de césped son aquellas donde predominan los prados y la vegetación herbácea perenne de escasa altura. Superficies selladas son áreas donde predominan los materiales impermeables o poco permeables, que no permiten el desarrollo de organismos, generalmente conforman senderos o grandes explanadas. La evaluación del comportamiento térmico de las plazas de la ciudad, mostró el efecto que produce la distribución, disposición y materialidad de las tres estructuras que la componen sobre el desempeño térmico de la misma y en relación con su entorno (5) (11).

Existen diferentes métodos en la literatura internacional para evaluar los beneficios que producen los espacios verdes a diferentes escalas. Los métodos más utilizados refieren a mediciones in situ donde mediante un conjunto de equipos y procedimientos adecuados se extraen datos de casos existentes (12) (5). Por otro lado la simulación numérica permite evaluar alternativas de diseño y pronosticar el comportamiento térmico de espacios aún no construidos. De este modo es posible cuantificar las consecuencias de las decisiones de diseño sobre el medio urbano y edilicio (13) (14). Entre las herramientas de simulación disponibles se encuentran: SUNtool, SOLENE, RayMan, URSOS (15), Autodesk Ecotect Analysis 2010 (16). De modo específico, el software ENVI-met, permite la evaluación dinámica de los fenómenos de transferencia de masa y energía a escala urbana, y ha demostrado ser un modelo solvente en términos de su capacidad para la representación adecuada de los fenómenos que ocurren en diferentes entornos urbanos; tomando como parámetro su grado de ajuste con las mediciones en campo. Actualmente, es uno de los programas más utilizados en la literatura internacional (17) y ha sido empleado en numerosas investigaciones en referencia al diseño urbano sustentable y la planificación de los espacios abiertos calles, plazas y parques (18) (19) (20) (21).

En este marco, el objetivo general del presente trabajo es detectar premisas de diseño que permitan mejorar las condiciones ambientales y la habitabilidad de las plazas urbanas en climas áridos. Para ello, se propone simular en ENVI-met 3.1 el comportamiento térmico de cada una de las estructuras
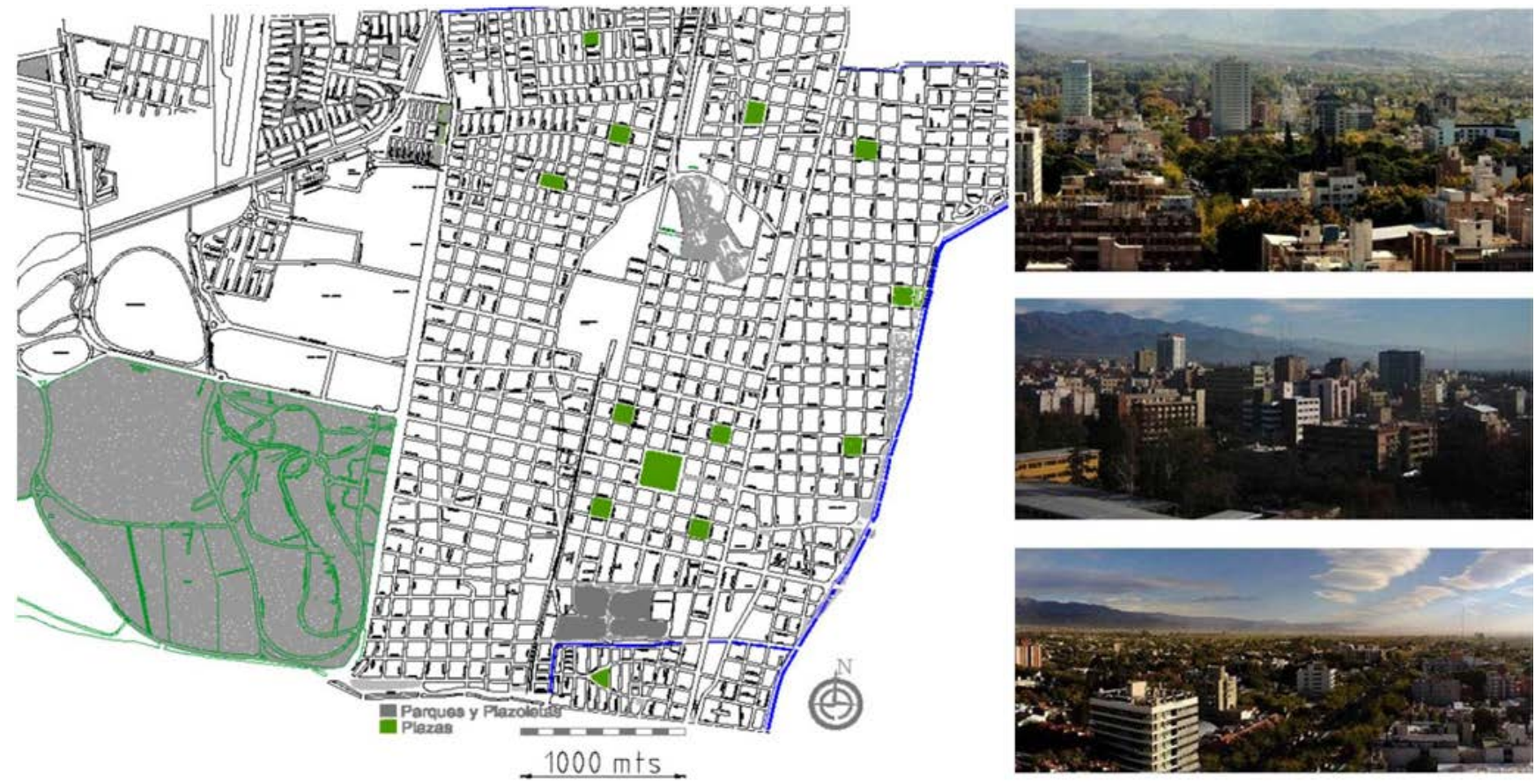

Figura 1. Características de la ciudad de Mendoza - Argentina. 
que componen las plazas -arbolado de gran porte, áreas de césped, superficies selladas- en forma aislada, es decir cuando constituyen el 100\% de la superficie de la misma. Y comparar el desempeño ambiental de cada una de estas estructuras puras con el de una plaza en condición real denominada "caso base" (donde las tres estructuras se encuentran combinadas). El fin último es determinar proporciones óptimas entre los distintos tipos de superficies vegetadas y selladas que componen una plaza, a fin de maximizar sus beneficios ambientales y propiciar el uso racional de los recursos naturales.

\section{METODOLOGÍA}

En la ciudad, como se dijo previamente, el diseño de las plazas existentes responde a una combinación, en distinta proporción, de tres estructuras predominantes: árboles de gran porte, áreas de césped y áreas de superficies selladas. Por este motivo sería muy difícil poder evaluar el desempeño ambiental de una plaza compuesta únicamente por sólo una de éstas estructuras mediante el monitoreo microclimático de un caso real. En este sentido, para obtener los resultados que permitan discutir el objetivo general del trabajo, es necesario recurrir a modelos computacionales -simuladores- que representan el comportamiento microclimático de un espacio construido con determinadas características. Por otra parte, la validez de los resultados que derivan de las simulaciones, se apoya en el grado de ajuste de las predicciones del modelo simulado con el comportamiento de un caso real monitoreado micro-climáticamente.

Por lo tanto el desarrollo metodológico del presente trabajo se divide en dos etapas, la primera destinada a la construcción y validación de un modelo teórico que represente el funcionamiento microclimático de un caso real considerado como caso base, y sobre el cual se harán las modificaciones necesarias para la construcción de los escenarios o casos hipotéticos. Y una segunda etapa que corresponde a la simulación de los casos hipotéticos a fin de evaluar la respuesta microclimática de las distintas alternativas de diseño propuestas.

En este apartado se describen los procedimientos necesarios para el desarrollo de la primera etapa que incluye: la caracterización climática de la ciudad de estudio y las características de la plaza seleccionada como caso base y su contexto de inserción, el monitoreo microclimático del caso real (Plaza Chile) seleccionado como caso base; su caracterización en términos de forma, materialidad y vegetación; y la validación o ajuste del modelo mediante la contrastación de los resultados obtenidos del caso base simulado con el caso real. Y los necesarios para la segunda etapa, que corresponde a la simulación de los tres casos hipotéticos propuestos: plaza compuesta exclusivamente por arbolado de gran porte -escenario 1-, plaza compuesta exclusivamente por superficies selladas -escenario 2-, plaza compuesta exclusivamente por áreas de césped -escenario $3-$.

\subsection{Modelo de Simulación Utilizado. ENVI-met 3.1}

ENVI-met es un programa de acceso gratuito desarrollado por Bruse, M. (1999) en el Instituto de Geografía de la Universidad de Mainz - Alemania (22), que busca reproducir los principales procesos atmosféricos que afectan al microclima sobre bases físicas fundadas, tales como las leyes de la dinámica y la termodinámica de fluidos (23). En relación al objetivo del presente trabajo, esta herramienta permite tener un enfoque integral de los efectos que producen los espacios abiertos vegetados en entornos urbanos, simulando la dinámica microclimática dentro de un ciclo diario. El modelo es estacionario y no hidrostático, pronostica todos los procesos de cambio, incluyendo flujos de viento, turbulencia, flujos de radiación, temperatura y humedad. Es posible una representación detallada de estructuras y materialidades urbanas complejas, es decir, edificios con diferentes formas y alturas, y propiedades ópticas de materiales. La vegetación es considerada no sólo como un obstáculo poroso al viento y a la radiación solar, sino también mediante la inclusión de los procesos fisiológicos de evapotranspiración y fotosíntesis. Se pueden seleccionar distintas tipologías de vegetación con propiedades específicas. El suelo también se considera como un volumen compuesto de varias capas y tipologías. La alta resolución espacial (hasta $0.5 \mathrm{~m}$ en sentido horizontal) y temporal (hasta 10 segundos) permiten una detallada lectura de los cambios micro climáticos. El modelo requiere un número limitado de entradas y proporciona un gran número de datos de salida. Además, calcula la temperatura media radiante (Tmr) que es una variable que condiciona la habitabilidad térmica de los espacios exteriores.

Algunas limitaciones han sido reportadas por González Viveros (2011) (24) quien discute las dificultades del modelo para representar correctamente el perfil forestal de especies locales debido a que la base de datos del programa contiene un número acotado de especies. Samaali et al. (2007) (25) comparó los resultados del cálculo del flujo de radiación en ENVImet con dos modelos validados, y mediciones de una experimentación en un campo de soja y determinó que ENVI-met simuló la transferencia radiativa de onda larga en el dosel de la vegetación con un buen nivel de ajuste. Sin embargo, reveló la debilidad de algunas hipótesis del modelo como la no atenuación de la radiación difusa de onda corta dentro de la vegetación. Krüger et al. (2011) (18) informaron que a $2.10 \mathrm{~m}$ sobre el nivel del suelo dentro del canal vial, las velocidades del viento predichas por ENVI-met eran consistentes con los datos de campo para velocidades del viento inferiores a 2.00 $\mathrm{m} / \mathrm{s}$, pero que el modelo tiende a sobrestimar las velocidades de viento por encima de $2.00 \mathrm{~m} / \mathrm{s}$. Comparando la temperatura del aire derivada de las estaciones meteorológicas locales en una ciudad desértica con los datos modelados, Chow et al. (2011) (26) concluyeron que ENVI-met tiende a subestimar la temperatura del aire durante el día pero sobreestimar la temperatura del aire durante la noche. Samaali et al. (2007) (25) atribuye estas subestimaciones y limitaciones en algunas de las variables a que el software fue desarrollado por un grupo de investigación que pertenece a latitudes medias, lo que puede implicar que aplicado a una ciudad que no se encuentra en esta latitud no se obtengan los resultados esperados, a menos que se encuentre alguna variable sistemática que se pueda ajustar. Pese a sus limitaciones es considerado una herramienta efectiva y con relevancia internacional para el análisis térmico y la evaluación del confort térmico del microclima dentro de las áreas urbanas (27) (28).

\subsection{Caracterización climática de la ciudad de estudio}

La ciudad de Mendoza se emplaza en el centro Oeste de la República Argentina, a $32^{\circ} 40^{\prime}$ Latitud Sur, $68^{\circ} 51^{\prime}$ Longitud Oeste y 750 m.s.n.m. (metros sobre el nivel del mar). El clima es BWk según la clasificación de Köppen, clima desértico con estepa fría / desierto (29). Se caracteriza por inviernos 
fríos y veranos calurosos. La temperatura promedio anual es de $16.50^{\circ} \mathrm{C}$, temperatura promedio máxima es de $24.50^{\circ} \mathrm{C}$ y la mínima de $9.60^{\circ} \mathrm{C}(30)$. Las lluvias son escasas 250.00 $\mathrm{mm}$ anuales promedio. La cantidad e intensidad de radiación solar es elevada debido a los numerosos días de cielo claro (2762 horas anuales de sol), radiación solar diaria en el verano $1022.00 \mathrm{~W} / \mathrm{m} 2$. La evapotranspiración potencial anual es de $1140.8 \mathrm{~mm}$. Los vientos son moderados y poco frecuentes, velocidad promedio: $11 \mathrm{~km} / \mathrm{h}$ con dirección sur- este (30), a excepción del viento Zonda, fenómeno se desarrolla eventualmente, entre los meses de mayo y octubre, es un viento seco y cálido, genera temperaturas de hasta $40.00^{\circ} \mathrm{C}$, sus ráfagas varían entre 65.00 a $120.00 \mathrm{~km} / \mathrm{h}$.

\subsection{Descripción del área simulada}

Como caso base se seleccionó la "Plaza Chile", por presentar características representativas que identifican las plazas en la ciudad de Mendoza en cuanto a: entorno edilicio, forma, diseño y materialidad, entre otros. Se ubica en el centro de la ciudad, en un contexto de media densidad edilicia (donde los edificios rondan entre los 3 pisos de altura y no superan los 6 pisos de altura), en una zona de uso comercial - mixta, tiene una superficie de 11995.28 m2. Forma parte del conjunto de espacios abiertos primarios de la ciudad (compuestos por una plaza central y cuatro plazas equidistantes a la misma). Respecto a organización interna de la plaza, consta con un núcleo central predominantemente semi-sellado, con la presencia de una fuente principal. Alrededor se sitúan áreas verdes con arboledas y césped, determinando los espacios respecto a un sistema radial. En cuanto a su materialidad presenta $48.72 \%$ de áreas selladas, 50.83\% de áreas verdes y 0.44\% de otras superficies. Muestra una relación verde/sellado de 1.05:1.

\subsection{Monitoreo estaciones fijas y determinación de día de simulación}

Para validar los resultados de las simulaciones es necesario ajustar los parámetros de simulación que requiere el modelo físico cargado en el programa EMVI-met con los datos medidos en un caso real que se elige como caso base.

Por ello, la Plaza chile fue monitoreada micro-climáticamente mediante estaciones fijas colocadas en el área de estudio.
Dichas mediciones se desarrollaron, durante la temporada de verano, utilizando estaciones fijas del tipo Ho8-003-02, con dos canales internos: temperatura y humedad relativa, registrando datos cada 15 minutos. Se midieron un total de 30 días (desde el 28 de diciembre de 2012 al 28 de enero de 2013). Los puntos de monitoreo fijo para la obtención de los datos micro-climáticos se situaron siguiendo el eje Norte - Sur, de la plaza (Ver figura 2).

Para la selección del día de análisis que luego será simulado, se analizaron los comportamientos de las curvas de temperatura del aire, y se evaluó estadísticamente el desempeño de las temperaturas máximas, mínimas y medias, junto a los niveles de radiación global y difusa (31). Del periodo monitoreado se seleccionaron dos días consecutivos el 11 y 12 de enero de 2013. Los días seleccionados para análisis son estables, con altos niveles de radiación solar, baja nubosidad, velocidad de viento y humedad, características representativas de un día típico de verano en Mendoza (32). Estadísticamente las condiciones meteorológicas del día seleccionado se replican para el $78 \%$ del total de días medidos (representatividad dentro del periodo monitoreado).

\subsection{Descripción del modelo físico para la simulación. Caracterización formal, material y de la vegetación presente en el caso base. (Plaza Chile)}

El área representada (simulada) consta de una superficie de $60000 \mathrm{~m}^{2}$ situándose la plaza en el centro. Conjuntamente con el relevamiento de la geometría del espacio y alturas de los edificios, se registró la materialidad, y los tipos de vegetación que componen la plaza, para una mayor precisión en la representación física del espacio simulado.

En lo que respecta a la materialidad se tuvo en cuenta cada una de las cuadras que conforman el entorno y el interior de la plaza, para hacer una ponderación de los materiales y asignarles las propiedades ópticas y térmicas de acuerdo a sus características. En el modelo se determinaron dos tipos de materiales de características diferentes que se incorporaron a la base de datos PROFILES.DAT y SOILS.DAT. Nombrados con las siglas V1 (destinados al entorno de la plaza) albedo 0.30 y emisividad 0.90; el modelo de suelo está compuesto
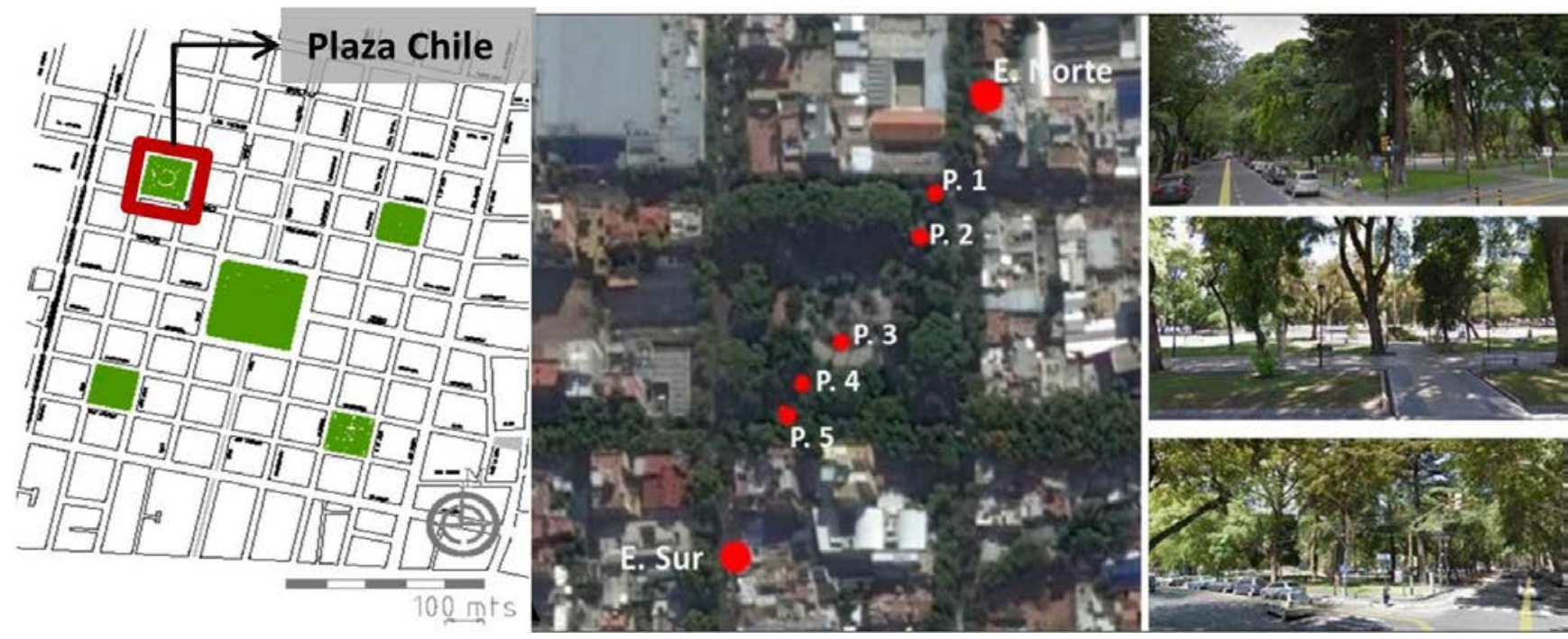

Figura 2. Área de estudio "plaza Chile” y puntos de monitoreo. 
por concreto, arena y arena arcillosa. Y los denominados V9, (representa los materiales utilizados en el interior de la plaza) con albedo 0.35 y emisividad o.90; el modelo de suelo está definido por granito, concreto y arena.

La vegetación se seleccionó a partir de conciliar las características de los forestales in situ con aquellos que ofrece el programa ENVI-met en su base de datos. Para representar el caso base (Plaza Chile) se tuvieron en cuenta cinco categorías: árboles densos de $20.00 \mathrm{~m}$ (Do), muy densos de 15.00 $\mathrm{m}$ ( Tb), livianos de 15.00 y $20.00 \mathrm{~m}$ (l1 y l2), árboles de tallo libre (Sc) y pastos, respecto a este último, se creó un nuevo elemento denominado $\mathrm{CH}$ con las características de las hierbas existentes pero con una altura de hasta $0.20 \mathrm{~cm}$, por lo general en nuestras plazas no encontramos pastos de mayor altura.

\subsection{Simulación del caso base. Datos y variables de ingreso}

Los datos de entrada al simulador se dividen en tres grupos: variables climáticas, variables geométricas y variables térmicas, las dos últimas descriptas en el punto anterior; requiriendo la determinación obligatoria de un número mínimo de variables que caracterizan las condiciones de borde. El modelo se simuló con una grilla 100 x 100 x 30, con una resolución del área de $3 \times 3 \times 3$ m, el número de grillas totales es de x: 93; y: 93; z: 30, debido a que la superficie de referencia es de $236 \times 236 \mathrm{~m}$.

Dentro del área simulada se colocaron 7 puntos monitores en total, cinco dentro de la plaza $\left(\mathrm{P}_{1}, \mathrm{P}_{2} ; \mathrm{P}_{3}, \mathrm{P}_{4} ; \mathrm{P}_{5}\right)$ y dos en el entorno de la misma (EN, ES) respetando el eje transversal Norte Sur. Los receptores para la obtención de los datos se situaron en coincidencia con los puntos de monitoreo fijo de la plaza (Ver Figura 3).

Las variables climáticas como la velocidad y dirección de viento a $10.00 \mathrm{~m}$ de altura, y la humedad relativa se tomaron de la estación "Mendoza Aeropuerto - SAME" (30). La temperatura de la atmósfera, y la humedad específica a $2500.00 \mathrm{~m}$ se obtuvo mediante datos de radio sondeos lanzados en el Aeropuerto de Mendoza en colaboración con la Universidad De Wyoming. La radiación solar de onda corta es un dato opcional para el programa. ENVI-met calcula la radiación solar (W/m2) mediante datos de localización geográfica previamente seleccionados, lo que le permite determinar la altura solar, azimut y flujo de radiación (directa, difusa y global). Con los valores de radiación solar calculados por el software y los valores medidos es posible ingresar un coeficiente de ajuste para hacer coincidir la curva de radiación simulada con la medida. Los rangos de ajuste válidos son de 0.50 (50\% del valor de ENVI-met no modificado) a 1.50 (150\%). En este estudio el coeficiente de ajuste utilizado fue 0.90 (90\%).

\subsection{Ajuste del modelo teórico con el caso real (Plaza Chile)}

Para validar los resultados que arrojan las simulaciones, es necesario ajustar los resultados de la simulación del modelo físico cargado para representar el funcionamiento del caso base (Plaza Chile) con los datos obtenidos del monitoreo microclimático de la plaza. Se realizó el ajuste del comportamiento de la curva de temperatura del aire simulada con la curva del aire medida correspondiente al día de análisis seleccionado.

Se probaron más de veinte escenarios de simulación, para alcanzar un nivel de ajuste que refleje las condiciones reales de la plaza. Se obtuvieron valores de $\mathrm{R}^{2}$ entre 0.89 a 0.96 . Tomando como referencia la literatura internacional (20) que alcanza un nivel de ajuste del orden de 0.63 a 0.77 en el presente trabajo se alcanza un nivel de ajuste muy bueno. Las diferencias entre temperaturas máximas oscilan entre $0.04^{\circ} \mathrm{C}$ a $4.79^{\circ} \mathrm{C}$ y las mínimas entre $0.14^{\circ} \mathrm{C}$ a $1.69^{\circ} \mathrm{C}$. En cuanto a las diferencias medias según el trabajo realizado por Song y Park (13) donde hace referencia al trabajo realizado por Park en su Ph.D se hallan diferencias de $7.00^{\circ} \mathrm{C}$ a $11.00^{\circ} \mathrm{C}$. En nuestro caso dichas diferencias se dan entre $0.40^{\circ} \mathrm{C}$ y $1.50^{\circ} \mathrm{C}$. Finalmente, a partir de la contrastación entre los resultados del ajuste obtenido a nivel internacional y los resultados obtenidos en nuestro caso, podemos justificar la selección del

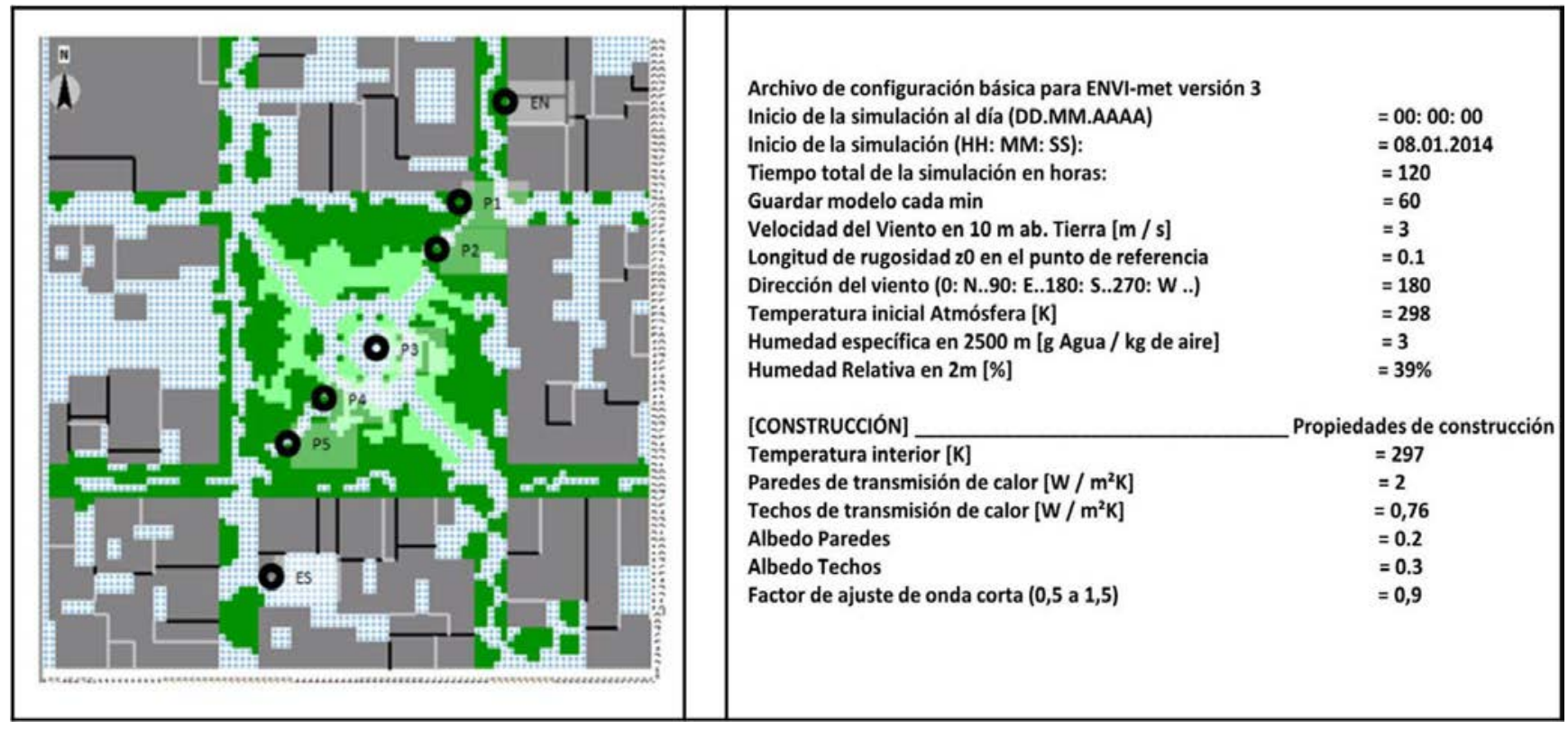

Figura 3. Puntos de monitoreo y configuración del área de simulada. 
modelo teórico como base física para evaluar el efecto de la modificación de distintas variables y su influencia sobre el comportamiento térmico de la plaza (ver figura 4).

\subsection{Simulación de casos Hipotéticos. Confección de escenarios}

A partir de las premisas consideradas para el desarrollo de esta investigación, se simularon tres casos compuestos al $100 \%$ por cada estructura, definida como: arbolado de gran porte, superficies selladas y áreas de césped. Los resultados del comportamiento ambiental de cada escenario hipotético simulado serán contrastados con los resultados del compor- tamiento ambiental del caso base simulado y ajustado previamente (Ver figura 5).

Este trabajo procura establecer el comportamiento ambiental de una plaza conformada por cada una de las estructuras al 100\%. De este modo pretende determinar los rangos máximos esperables de beneficios térmico - ambientales que cada estructura puede proporcionar al espacio urbano.

\section{RESULTADOS}

Se analiza el comportamiento de las tres estructuras en forma individual, definidas como arbolado de gran porte, áreas

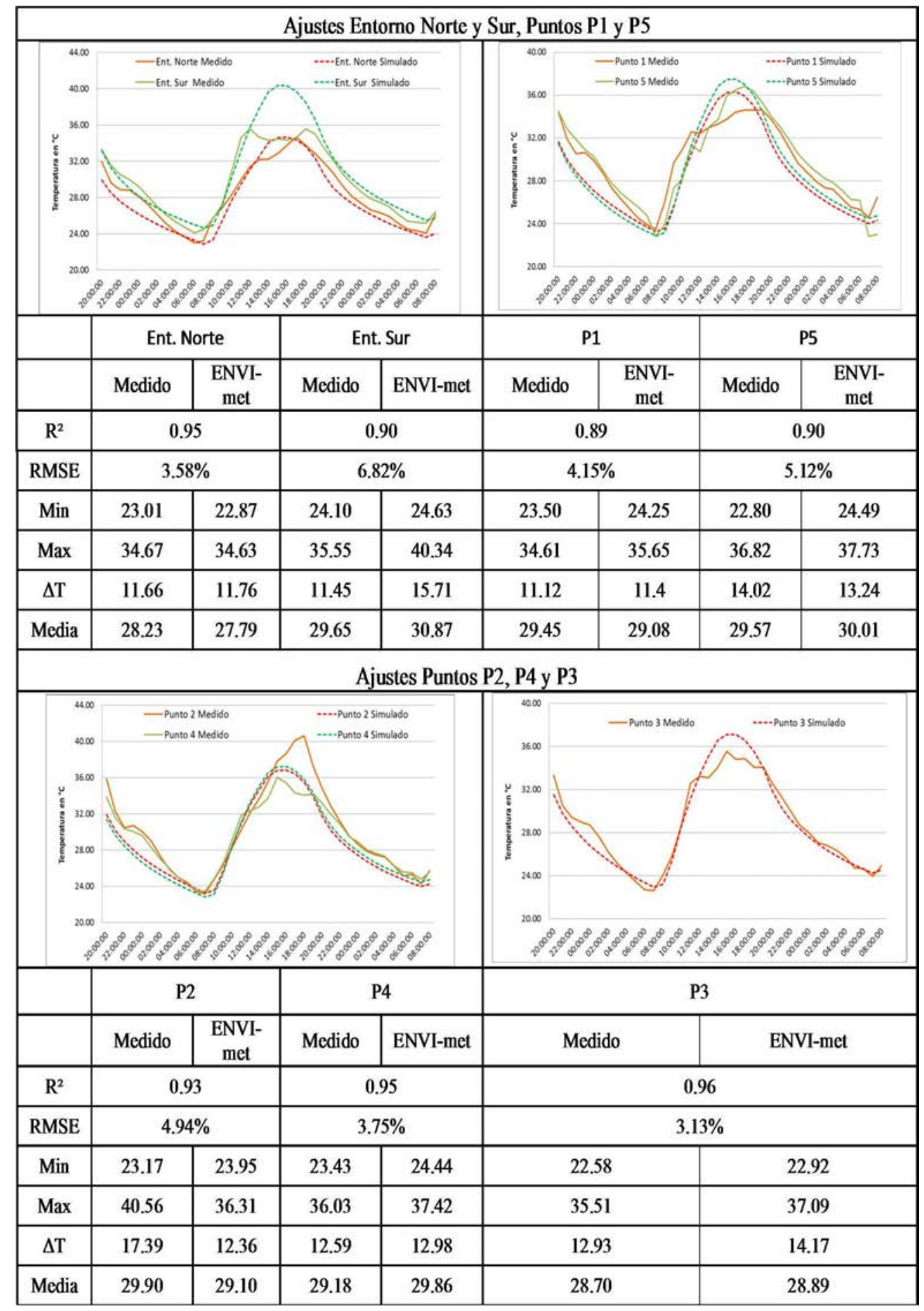

Figura 4. Ajustes Entornos Norte y Sur, punto 1 y punto 5, punto 2, punto 4 y punto 3. 


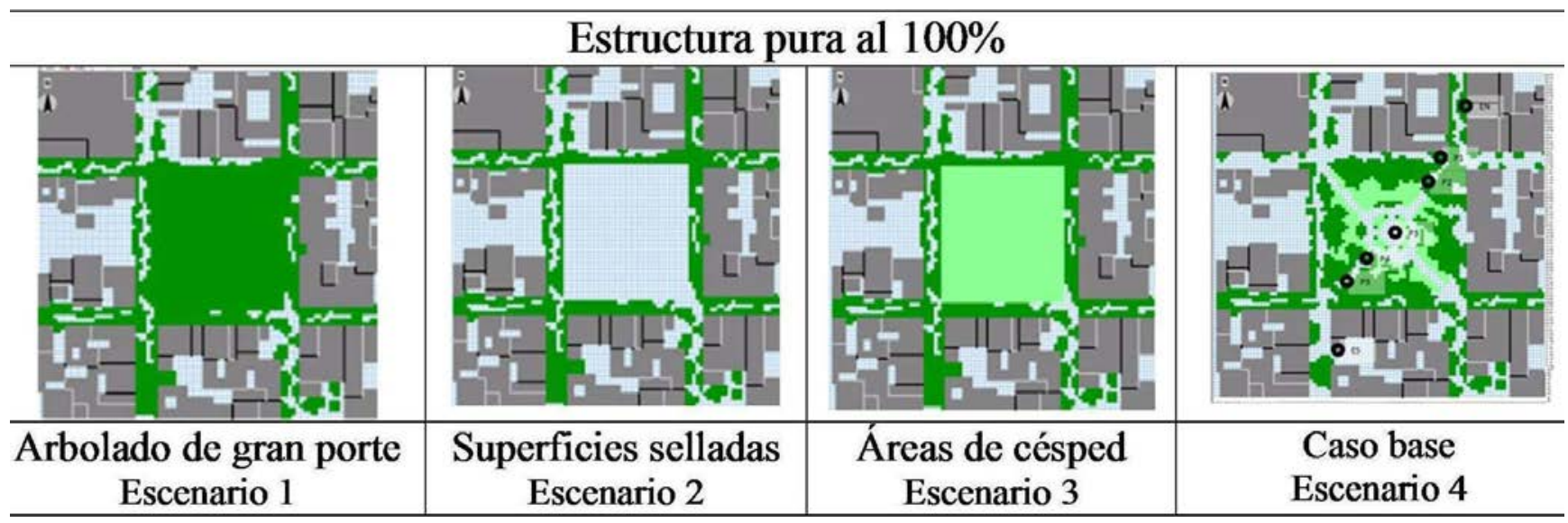

Figura 5. Esquema de simulación. Arbolado de gran porte, superficies selladas, áreas de césped al $100 \%$ y Caso base.

de césped, superficies selladas al 100\%. Posteriormente se analiza el comportamiento microclimático de cada estructura respecto al caso base (simulado y ajustado con el caso real). El objetivo final es encontrar una combinación óptima de estructuras asociadas al diseño de la plaza.

Se analizan datos de temperatura del aire (Ta), temperaturas medias radiantes (Tmr) y velocidad de viento $(\mathrm{Vv})$ a $1.50 \mathrm{~m}$ de altura, para los cuatro escenarios. Se diferencian respecto a su comportamiento diario discriminado en dos períodos de: calentamiento (8:00 a.m. a 7:00 p.m.) y enfriamiento (8:00 p.m. a 7:00 a.m.).

Respecto a los datos obtenidos en relación a la temperatura del aire, la estructura más eficiente es el arbolado de gran porte. Presenta temperaturas mínimas de $24.00^{\circ} \mathrm{C}$, máximas $35.50^{\circ} \mathrm{C}$ y promedios de $28.50^{\circ} \mathrm{C}$, esto se debe al beneficio de la sombra por sobre la materialidad. Las áreas de césped (materialidad verde de escasa altura) registra temperaturas superiores respecto al arbolado de gran porte de $2.00^{\circ} \mathrm{C}$ más respecto a las máximas $\left(37.50^{\circ} \mathrm{C}\right), 1.00^{\circ} \mathrm{C}$ más respecto a las mínimas $\left(25.00^{\circ} \mathrm{C}\right)$ y diferencias de $1.50^{\circ} \mathrm{C}$ respecto a la temperatura promedio diaria $30.00^{\circ} \mathrm{C}$ (ver figura 6). La estructura de peor desempeño térmico, corresponde a superficies selladas al 100\%, condición representativa de plazas europeas y tendencia predominante en el diseño de espacios abiertos urbanos, sus temperaturas máximas alcanzan los $39.00^{\circ} \mathrm{C}$, la temperatura mínima presenta un valor de $25.50^{\circ} \mathrm{C}$ y su temperatura promedio es de $31.00^{\circ} \mathrm{C}$ (ver figura 6).

Para el periodo de calentamiento (durante el día) el promedio de las temperaturas para la estructura analizada como más favorable es el escenario 1 - arbolado de gran porte - sus temperaturas alcanzan los $32.00^{\circ} \mathrm{C}$ y como más desfavorable para el escenario 3 - superficies selladas $-35.00^{\circ} \mathrm{C}$. Este periodo es considerado como el periodo de mayor disconfort térmico tanto en las plazas urbanas como en los espacios al aire libre, ya que son espacios de transición dentro de la trama

Durante el periodo de enfriamiento (la noche) las temperaturas promedio alcanzan los $26.50^{\circ} \mathrm{C}$ en el escenario 1- arbolado de gran porte al $100 \%$ y $28.50^{\circ} \mathrm{C}$ en el escenario 3 - superficies selladas al 100\%. Es decir entre la mejor y la peor de las configuraciones se registran diferencias de temperaturas de $3.50^{\circ} \mathrm{C}$ respecto de las máximas y $1.50^{\circ} \mathrm{C}$ respecto de las mínimas. Sin embargo en términos de habitabilidad las diferencias entre las temperaturas promedio de ambas estructuras son de $3.00^{\circ} \mathrm{C}$ durante el periodo de calentamiento y $2.00^{\circ} \mathrm{C}$ durante el enfriamiento (ver figura 6).

Según Givoni (33), los límites de la zona de confort a humedades bajas y medias son independientes del nivel de humedad, ya que el confort de las personas en estado sedentario y con ropa común no se ve afectado. A humedades más altas los efectos de la temperatura y la humedad están interrelacionados y el límite superior de temperatura disminuye con una mayor humedad. Para nuestro clima, los límites de temperatura sugeridos cuando el aire está quieto son de $20.00^{\circ} \mathrm{C}$ a $27.00^{\circ} \mathrm{C}$ en verano. Para las personas que viven en países en desarrollo y calurosos el autor sugiere elevaciones de aproximadamente $2.00^{\circ} \mathrm{C}$ en el límite superior de temperatura, teniendo en cuenta la aclimatación como resultado de vivir en edificios sin aire en un clima caliente. Este límite de temperatura superior es aplicable a bajos niveles de humedad (por debajo de un contenido de vapor de $12.00 \mathrm{~g} / \mathrm{kg}$ ). Esta situación es coincidente con la situación microclimática de la ciudad de Mendoza.

De acuerdo a esto, y con los valores de temperaturas promedio obtenidas, podríamos decir que durante el periodo de enfriamiento todas las configuraciones se encontrarían dentro del rango de confort extendido, sin embargo la configuración de arbolado de gran porte es la única que se encuentra dentro del rango de confort de temperatura convencional $-26.50^{\circ} \mathrm{C}-$. En el periodo de calentamiento todas se alejan de la condición de confort, siendo el arbolado de gran porte quien presenta las menores temperaturas promedio $-32.00^{\circ} \mathrm{C}-$.

Si se analiza el comportamiento del entorno radiante expresado por su temperatura media radiante, (máxima, mínima y promedio) vemos que la configuración más ventajosa en términos de la habitabilidad es el escenario 1 - arbolado de gran porte - tanto para el periodo de enfriamiento (temperatura máxima $23.50^{\circ} \mathrm{C}$ ) como para el periodo de calentamiento (temperatura máxima $35 \cdot 50^{\circ} \mathrm{C}$ ).

Durante el periodo más demandante - periodo de calentamiento- (en lo que respecta a los efectos de la radiación solar), se observa que las diferencias entre la configuración del escenario 1 - arbolado de gran porte al 100\%- configuración más ventajosa; (Tmr máxima $35 \cdot 50^{\circ} \mathrm{C}$ ) y la condición más desfavorable, Escenario 3 -superficies selladas al 100\%- (Tmr máxima $69.00^{\circ} \mathrm{C}$ ), alcanzan diferencias de los $33.50^{\circ} \mathrm{C}$ de para las Tmr máximas y $31.50^{\circ} \mathrm{C}$ para las Tmr promedio. (Ver figura 6). 


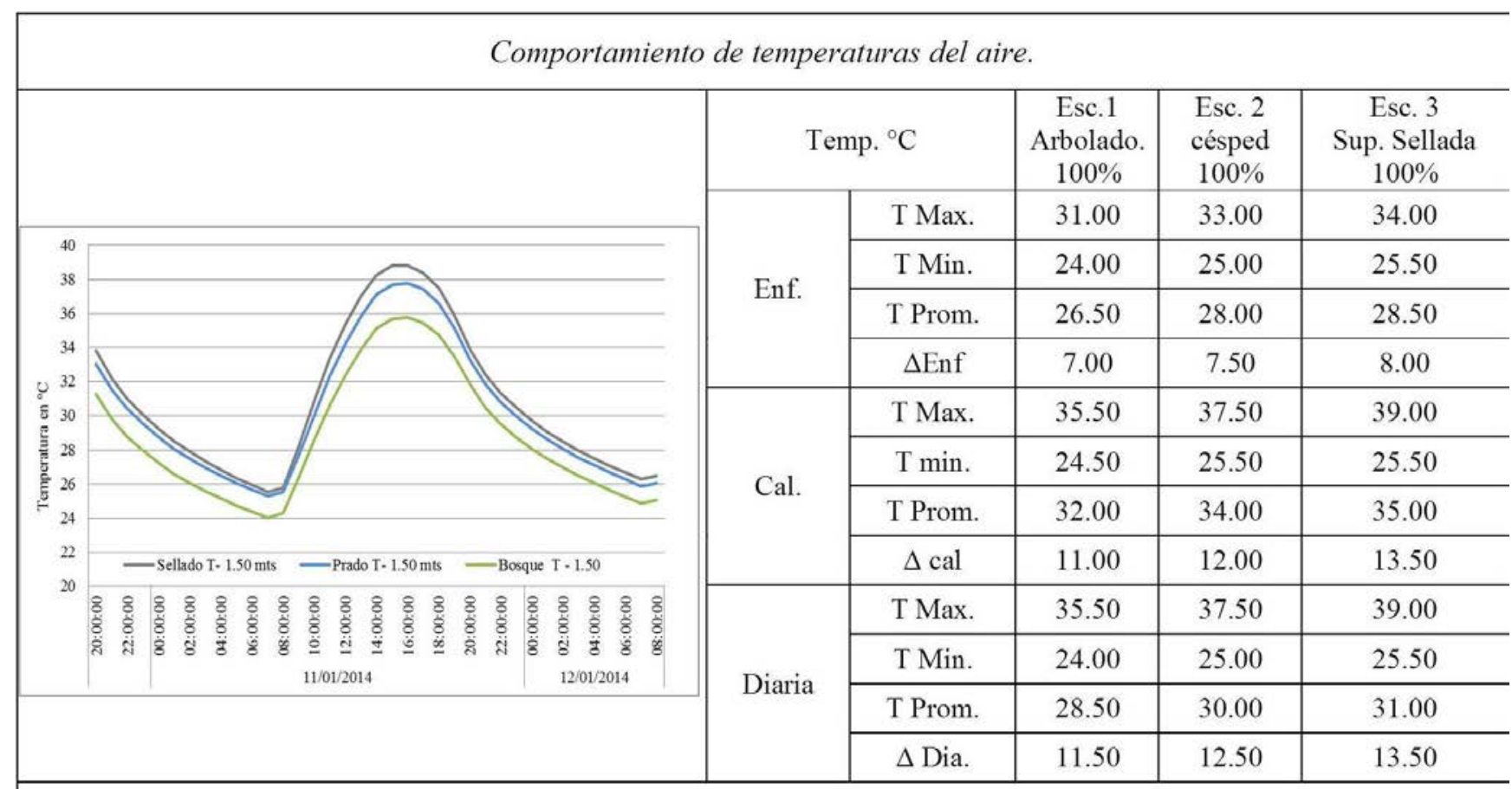

Comportamiento de temperaturas medias radiante.

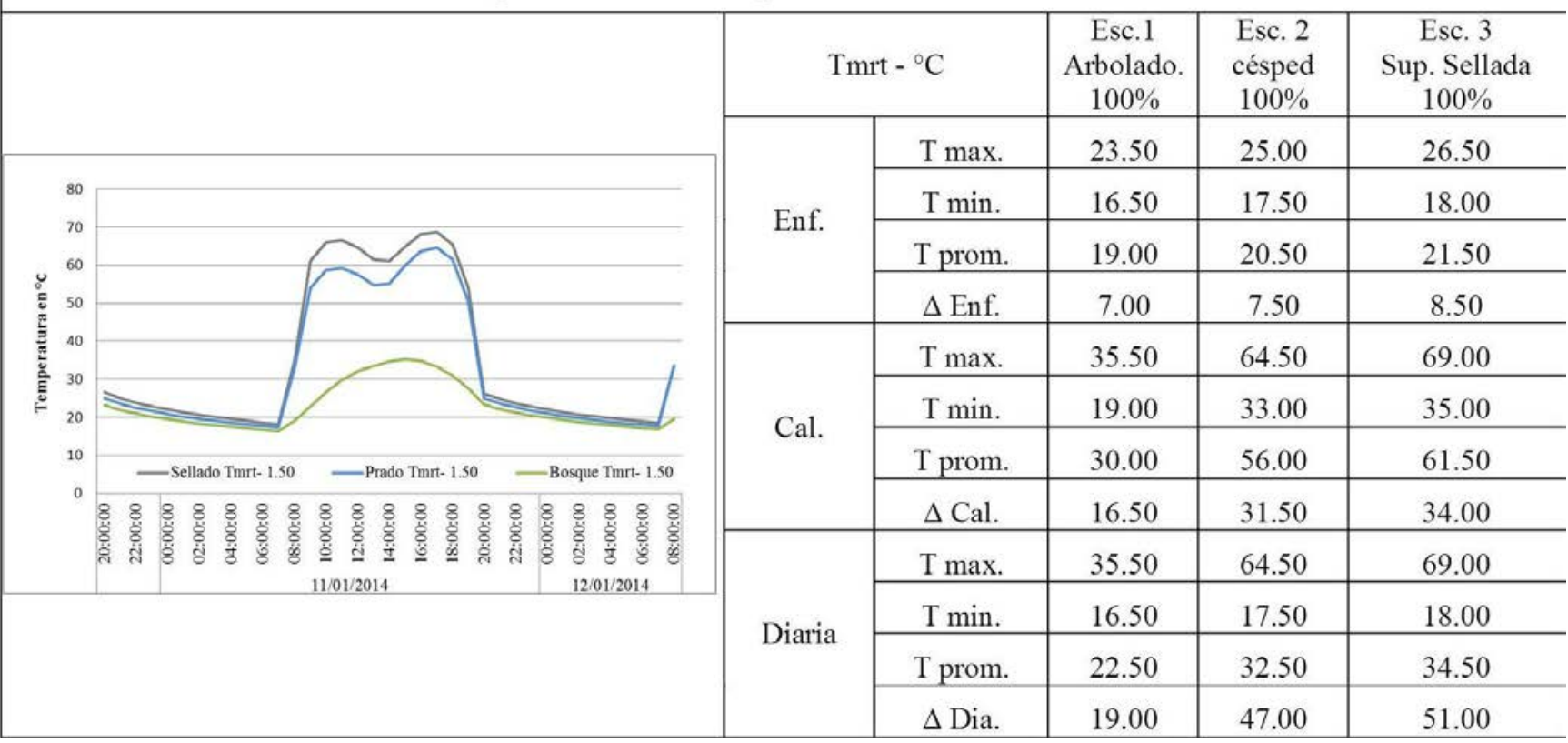

Figura 6. Comportamiento temperaturas del aire temperaturas medias radiante. Arbolado de gran porte, superficies selladas, áreas de césped al 100\%.

En lo que respecta al escenario 2 (áreas de césped al 100\%) tanto en el periodo de enfriamiento como en el periodo de calentamiento, presenta temperaturas intermedias respecto al escenario más beneficioso (arbolado de gran porte al 100\%) y al más desfavorable (superficies impermeables 100\%), su Tmr máxima es $64.50^{\circ} \mathrm{C}$ superior a la del arbolado de gran porte y su Tmr promedio es $32.50^{\circ} \mathrm{C}$ (Ver figura 6).

A partir de los resultados analizados podríamos decir que las diferencias de temperatura del aire encontradas entre la mejor y peor configuración (áreas de césped al 100\%) versus (superficies impermeables al 100\%) -es de $3.50^{\circ} \mathrm{C}$ para el caso de las máximas, $2.50^{\circ} \mathrm{C}$ para el caso de los promedios y $1.50^{\circ} \mathrm{C}$ para el caso de las mínimas- esto muestra que las características de la configuración de la plaza afectan en mayor magnitud el valor de la temperatura del aire durante el día. Respecto a la Temperatura media radiante (Tmr), variable que condiciona severamente la habitabilidad de los espacios, se ve aún más severamente afectada por la configuración de la plaza que la temperatura del aire.

La tabla 1 presenta los datos del desempeño térmico de las tres estructuras, comparando en el caso base con los tres escenarios simulados al $100 \%$. Los resultados de esta comparación ponen énfasis en que el escenario 1 (arbolado de gran porte al $100 \%$ ) es la única de las estructuras que ocupando el 100\% del espacio de la plaza mejora su desempeño térmico. El resto de los escenarios, escenario 3, áreas de césped al 100\% y escenario 2 , superficies selladas al $100 \%$ que empeoran el desempeño de la plaza cuando no están combinadas (Ver tabla 1 y figura 7). 
Tabla 1. Temperatura del aire, comparación estructura de arbolado de gran porte, superficies selladas, áreas de césped al 100\% versus caso base.

\begin{tabular}{|c|c|c|c|c|c|c|c|c|c|c|}
\hline \multirow{2}{*}{\multicolumn{2}{|c|}{$\mathbf{T a}\left[{ }^{\circ} \mathbf{C}\right]$}} & \multicolumn{2}{|c|}{$\begin{array}{c}\text { Esc. } 1 \\
\text { Arbolado }\end{array}$} & \multirow{3}{*}{$\begin{array}{c}\Delta \\
\text { Arbolado } \\
\text { Base - } \\
\text { Simulado } \\
1.50\end{array}$} & \multicolumn{2}{|c|}{$\begin{array}{l}\text { Esc. } 2 \\
\text { césped }\end{array}$} & \multirow{3}{*}{$\begin{array}{c}\Delta \\
\text { Césped } \\
\begin{array}{c}\text { Base - Si- } \\
\text { mulado }\end{array} \\
-1.00\end{array}$} & \multicolumn{2}{|c|}{$\begin{array}{c}\text { Esc. } 3 \\
\text { Sup. Sellada }\end{array}$} & \multirow{3}{*}{$\begin{array}{c}\Delta \\
\text { Sellado } \\
\text { Base - } \\
\text { Simulado } \\
-2.00\end{array}$} \\
\hline & & \multirow{2}{*}{$\begin{array}{c}\text { Caso base } \\
32.50 \\
\end{array}$} & \multirow{2}{*}{$\begin{array}{c}\begin{array}{c}\text { Simulado } \\
\mathbf{1 0 0 \%}\end{array} \\
31.00 \\
\end{array}$} & & \multirow{2}{*}{$\begin{array}{c}\text { Caso base } \\
32.00 \\
\end{array}$} & \multirow{2}{*}{\begin{tabular}{|c|}
$\begin{array}{c}\text { Simulado } \\
\mathbf{1 0 0} \%\end{array}$ \\
33.00 \\
\end{tabular}} & & \multirow{2}{*}{$\begin{array}{c}\text { Caso base } \\
32.00\end{array}$} & \multirow{2}{*}{\begin{tabular}{|c|}
$\begin{array}{c}\text { Simulado } \\
\mathbf{1 0 0 \%}\end{array}$ \\
34.00 \\
\end{tabular}} & \\
\hline$\stackrel{2}{=}$ & T Máx. & & & & & & & & & \\
\hline$\cdot \stackrel{\bar{\Xi}}{\Xi}$ & T Mín. & 24.50 & 24.00 & 0.50 & 24.50 & 25.00 & -0.50 & 24.00 & $25 \cdot 50$ & -1.50 \\
\hline . & T Prom. & 27.50 & 26.50 & 1.00 & 27.00 & 28.00 & -0.50 & 27.00 & 28.50 & -1.50 \\
\hline 玒 & $\Delta \mathrm{T}$ & 8.00 & 7.00 & 1.00 & 7.50 & 8.00 & -0.50 & 8.00 & 8.50 & -0.50 \\
\hline \multirow{4}{*}{ 串 } & T Máx. & 37.50 & $35 \cdot 50$ & 2.00 & 37.50 & $37 \cdot 50$ & 0.00 & 37.00 & 39.00 & -2.00 \\
\hline & T Mín. & 24.50 & 24.50 & 0.00 & 24.50 & $25 \cdot 50$ & -1.00 & 24.50 & $25 \cdot 50$ & -1.00 \\
\hline & T Prom. & 33.50 & 32.00 & 1.50 & 33.50 & 34.00 & -0.50 & 33.00 & 35.00 & -2.00 \\
\hline & $\Delta \mathrm{T}$ & 13.00 & 11.00 & 2.00 & 13.00 & 12.00 & 1.00 & 12.50 & 13.50 & -1.00 \\
\hline \multirow{4}{*}{ } & T Máx. & 37.50 & $35 \cdot 50$ & 2.00 & 37.50 & 37.50 & 0.00 & 37.00 & 39.00 & -2.00 \\
\hline & T Mín. & 24.50 & 24.00 & 0.50 & 24.50 & 25.00 & -0.50 & 24.00 & $25 \cdot 50$ & -1.50 \\
\hline & T Prom. & 29.50 & 28.50 & 1.00 & 29.50 & 30.00 & -0.50 & 29.00 & 31.00 & -2.00 \\
\hline & $\Delta \mathrm{T}$ & 13.00 & 11.50 & 1.50 & 13.00 & 12.50 & 0.50 & 13.00 & 13.50 & -0.50 \\
\hline
\end{tabular}
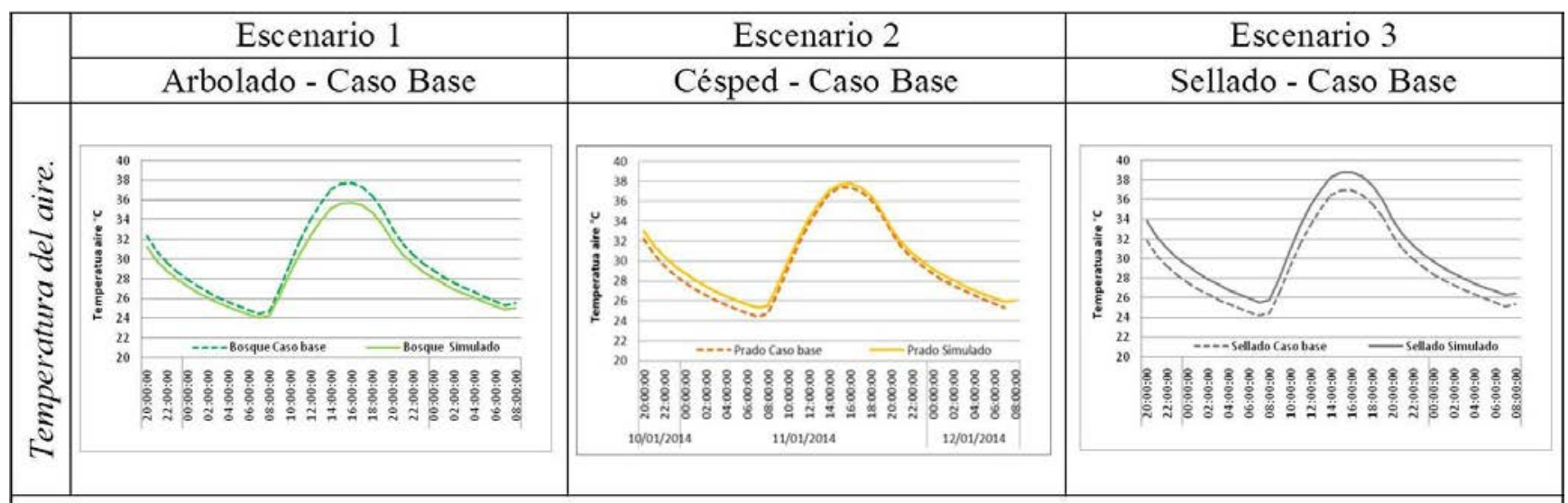

Comportamiento de la temperatura del aire. Estructura dentro del caso base versus al $100 \%$.
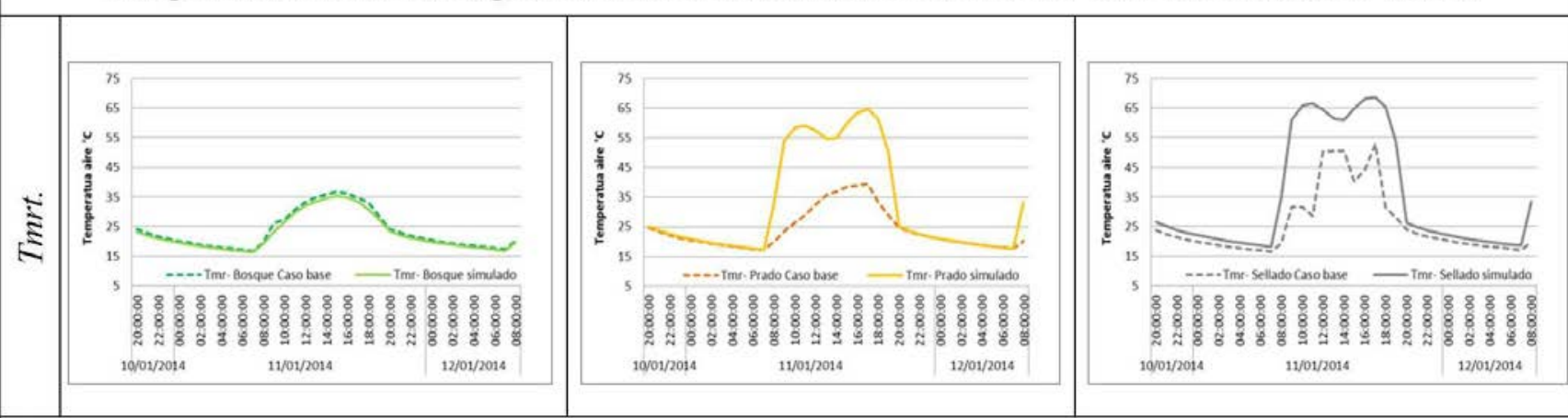

Comportamiento de la Tmrt. Estructura dentro del caso base versus al 100\%.

Figura 7. Comportamiento de las temperaturas del aire y Tmrt. Comparación estructura al 100\% versus caso base.

La misma tendencia de comportamiento se observa al contrastar las Tmr. La estructura que tiene el entorno radiante más afectado cuando ocupa el 100\% de la plaza corresponde a las áreas de césped. Cuando esta estructura está combinada con otras que producen sombras su comportamiento mejora y se asemeja al desempeño radiante del arbolado de gran por- te. Si la estructura se encuentra pura su entorno radiante se asemeja al del espacio sellado (Ver Tabla 2 y Figura 7).

Finalmente se observa, que la única estructura que mejora el desempeño térmico y radiante de la plaza cuando se encuentra al $100 \%$ es el arbolado de gran porte. De lo discutido pre- 
Tabla 2. Temperaturas medias radiantes. Estructura de arbolado de gran porte, superficies selladas, áreas de césped al 100\% versus caso base.

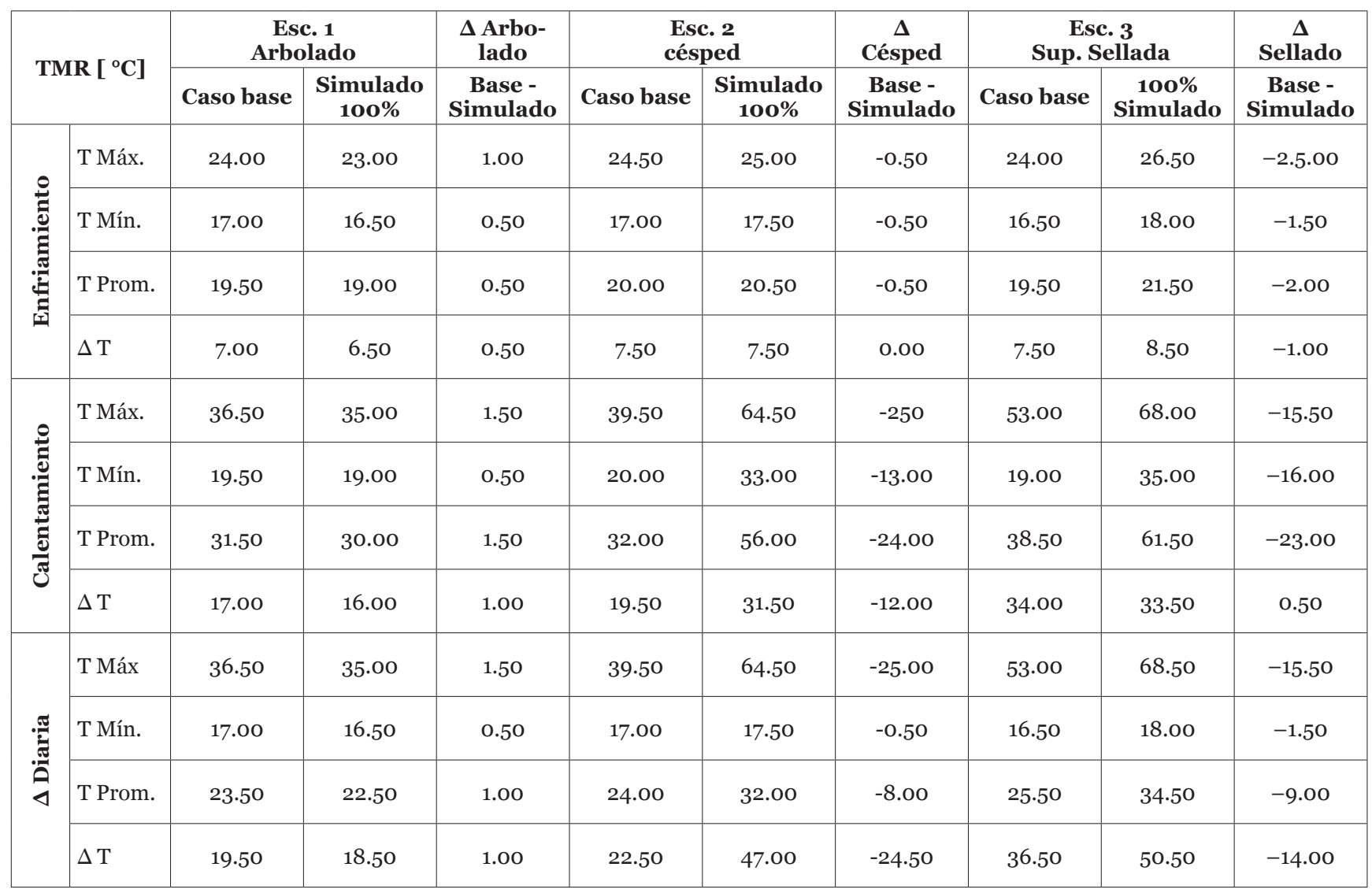

viamente, la configuración de plaza boscosa parecería ofrecer los mejores resultados en términos del desempeño térmico y ambiental, es decir mejores condiciones de habitabilidad durante el día y menores temperaturas del aire durante la noche. Permite generar nodos fríos dentro de la trama urbana y mejorar sus condiciones de enfriamiento colaborando con la mitigación de la isla de calor urbana.

Sin embargo de acuerdo a la funcionalidad de la plaza dentro de la trama urbana, es necesario garantizar su transitabilidad, es por ello que es inevitable la presencia de estructuras selladas. Por esta razón la próxima etapa de investigación va en busca de una relación óptima entre la estructura verde que ha demostrado ofrecer la mejor eficiencia ambiental -arbolado de gran porte- y la estructura sellada.

Otra variable que resulta necesario analizar es la velocidad del viento. Si bien la ciudad de Mendoza presenta baja frecuencia e intensidad a escala peatonal este recurso se encuentra condicionado por la geometría del cañón urbano y la intensa forestación característica de la ciudad. Determinar una tendencia de comportamiento sobre este recurso resulta necesario ya que contribuye en menor escala a potenciar el enfriamiento de la ciudad y cuando su magnitud se incrementa su impacto sobre las condiciones térmicas y de confort del espacio son muy importantes.

La figura 8A muestra el perfil de velocidad de viento a las 7 a.m. (línea continua) y 4 p.m. (línea de trazo) a diferentes alturas dentro de la plaza. Y la figura 8B muestra el comportamiento del perfil de viento en el entorno de la plaza para cada una de las configuraciones analizadas.
Si observamos los perfiles de viento del caso base simulado -Plaza Chile- donde las estructuras se encuentran combinadas). A nivel peatonal (1.50 m de altura), este caso presenta las menores velocidades de viento, oscilando su valor entre 0.80 y $1.00 \mathrm{~m} / \mathrm{s}$. Por otro lado la configuración que ofrece mayores velocidades de viento a esa altura es la de plaza con superficies impermeables al 100\% registrando valores dentro de un rango 1.50 y $1.70 \mathrm{~m} / \mathrm{s}$ (ver figura 8 ).

Los escenarios de configuraciones puras - arbolado de gran porte, áreas de césped y superficies impermeables al 100\%presentan siempre mayores velocidades de viento a baja altura (menor a $4 \mathrm{~m}$ ) que el caso base (donde se combinan las tres estructuras). Sin embargo las diferencias por debajo de esa altura no superan los $0.80 \mathrm{~m} / \mathrm{s}$. Las máximas diferencias entre los cuatro escenarios se registran a la altura de la copa del forestal -entre los 10.00 y $15.00 \mathrm{~m}$. A esa altura el escenario 3 (áreas de césped al 100\%) presentan velocidades de viento que superan hasta en $1.40 \mathrm{~m} / \mathrm{s}$ al escenario 1 (arbolado de gran porte al 100\%). Por encima de la altura de la copa de forestal $(25.00 \mathrm{~m})$ los perfiles tienden a juntarse, siendo mayor la velocidad de viento correspondiente al escenario 1 (arbolado de gran porte al 100\%) y menor en el escenario 3 (superficies impermeables al 100\%), donde las diferencias son del orden de $0.50 \mathrm{~m} / \mathrm{s}$ (Ver figura 8A).

Si analizamos el comportamiento de los entornos de cada uno de los escenarios evaluados, vemos que por debajo de los $10.00 \mathrm{~m}$ de altura, el comportamiento de los perfiles de viento es semejante, con valores del orden de $0.50 \mathrm{~m} / \mathrm{s}, \mathrm{y}$ a partir de los $10.00 \mathrm{~m}$ hacia arriba comienza a diferenciarse y acelerarse. Por encima de los $25.00 \mathrm{~m}$ se registran diferen- 


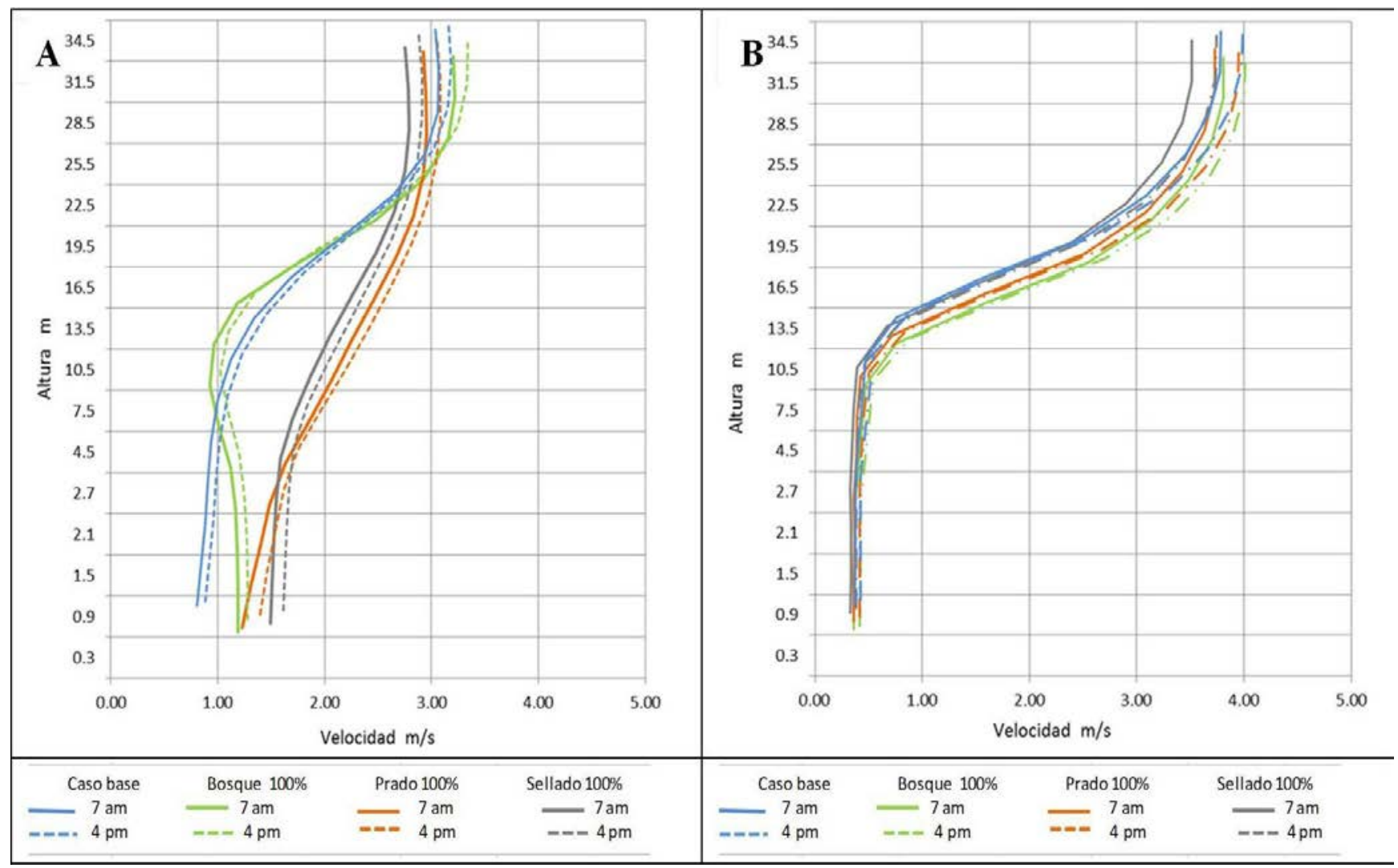

Figura 8. Perfil de viento (m/s) durante la hora de mínima y máxima temperatura (7 am y 4 pm).

cias del orden del $0.50 \mathrm{~m} / \mathrm{s}$ entre la el escenario 3 (plaza con superficies impermeables al 100\%) que muestra velocidades de viento del orden de los $3.00 \mathrm{~m} / \mathrm{s}$ y el escenario 1 (arbolado de gran porte al 100\%) que muestra velocidades del orden de $3.60 \mathrm{~m} / \mathrm{s}$.

A partir de los resultados encontrados podríamos decir que el incremento de la rugosidad asociada a la existencia del tronco y las copas de los árboles en las estructuras boscosas a nivel peatonal en la plaza significa disminuciones de la velocidad del viento que no superan los $0.80 \mathrm{~m} / \mathrm{s}$. Sin embargo, por encima de los $25.00 \mathrm{~m}$, el arbolado de gran porte al 100\% presenta las mayores velocidades de viento tanto en el contexto de la plaza como en su entorno mejorando de esta forma las condiciones de ventilación de la ciudad.

\section{CONCLUSIONES}

Este trabajo tuvo por objeto identificar la estructura pura más eficiente desde el punto de vista de su comportamiento térmico, la habitabilidad del espacio y su impacto en las posibilidades de enfriamiento de la ciudad. A los efectos de determinar la estructura más eficiente se simularon las distintas estructuras identificadas en las plazas en trabajos anteriores - arbolado de gran porte, superficies impermeables y áreas de césped en su condición pura (100\%). La configuración de la plaza con arbolado de gran porte al $100 \%$ ofrece los mejores resultados en términos del desempeño térmico y ambiental de la plaza, es decir mejores condiciones de habitabilidad durante el día y menores temperaturas del aire durante la noche. Permite generar nodos fríos dentro de la trama urbana y mejorar sus condiciones de enfriamiento colaborando con la mitigación de la isla de calor urbana.
Las diferencias de temperatura del aire encontradas entre la mejor y peor configuración son del orden de $3.50^{\circ} \mathrm{C}$ para el caso de las máximas, $2.50{ }^{\circ} \mathrm{C}$ para el caso de las promedio y $1.50^{\circ} \mathrm{C}$ para el caso de las mínimas. Estos resultados muestran que la configuración de la plaza en términos de estructuras afecta principalmente la temperatura del aire durante el día.

También se pudo establecer que la temperatura media radiante, que condiciona la habitabilidad térmica de los espacios es influenciada por la configuración de la plaza aún más severamente que la temperatura del aire, presentando entre la mejor y peor configuración diferencias del orden $33.50^{\circ} \mathrm{C}$ en las Tmr máximas.

La única estructura que mejora el desempeño térmico de la plaza cuando se encuentra pura es la de arbolado de gran porte. El incremento de la rugosidad debido a la existencia del tronco y las copas de los árboles en las estructuras boscosas a nivel peatonal disminuye la velocidad del viento hasta 0.80 $\mathrm{m} / \mathrm{s}$, sin embargo por encima de los 25.00 m esta estructura incrementa las mayores velocidades de viento tanto en el contexto de la plaza como en su entorno mejorando las condiciones de ventilación de la ciudad.

De lo descrito se desprende que desde el punto de vista ambiental las plazas debieran de conformar espacios urbanos con arbolado de gran porte distribuidos en la trama. Sin embargo los requerimientos funcionales de la plaza plantean la necesidad de contar con espacios de circulación peatonal y ámbitos ceremoniales, es por ello necesario la presencia de estructuras selladas. En futuros trabajos se evaluará la relación óptima entre ambas estructuras que permitan maximizar las prestaciones ambientales de la plaza sin comprometer sus prestaciones funcionales. 


\section{REFERENCIAS}

(1) Bowler, D., Buyung-Ali, L., Knight, T., Pullin, A. (2010) Urban greening to cool towns and cities: A systematic review of the empirical evidence. Landscape and Urban Planning. 97 (3):147-155. http://dx.doi.org/10.1016/j.landurbplan.2010.05.006

(2) Lafortezzaa, R., Carrusc, G., Sanesia , G., Davies C. (2009) Benefits and well-being perceived by people visiting green spaces in periods of heat stress. Urban Forestry \& Urban Greening 8: 97-108

(3) Carrus, G., Scopelliti M., Lafortezza R, Colangelo, G., Ferrini F., Salbitano F., Agrimi M., Portoghesi L., Semenzato P., Sanesi G. (2015) Go greener, feel better. The positive effects of biodiversity on the well-being of individuals visiting urban and peri-urban green areas Landscape and Urban Planning 134: 221-228

(4) Ruiz, A., Correa, E., Cantón, A., (2015) Incidencia de la selección de la especie forestal en el confort térmico de cañones urbanos de zonas áridas: el caso de Mendoza Argentina. Revista Urbano 32: 60 - 69 ISSN 0717-3997 / 0718-3607.

(5) Stocco, S., Cantón, M. A., Correa, E. (2013). Evaluación de las condiciones térmicas de verano y eficiencia ambiental de distintos diseños de plazas urbanas en Mendoza, Argentina. Hábitat Sustentable 3: 19-34.

(6) Shah, Md., Atiqul, Haq. (2011) Urban Green Spaces and an Integrative Approach to Sustainable Environment. Journal of Environmental Protection, 2: 601-608 doi:10.4236/jep.2011.25069.

(7) Lee, A.C.K., Maheswaran, R. (2010) The health benefits of urban green spaces: a review of the evidence. Journal of Public Health 33 (2): 212 -222 doi:10.1093/pubmed/fdqo68.

(8) Correa, E., Martinez, C., Lesino, G., De Rosa, C., Cantón, M. A., (2006). Impact of Urban Parks on the Climatic Pattern of Mendoza's Metropolitan Area, in Argentina. Conference on Passive and Low. Energy Architercure (PLEA 2006). Switzerland, September 2006.

(9) Stocco, S., Cantón, M. A., Correa, E. (2013). Condiciones térmicas y beneficios ambientales de distintas configuraciones de plazas urbanas en zonas áridas. Reunión de Trabajo de la Asociación Argentina de Energías Renovables y Medio Ambiente 1:05.61-05.68 ISBN 978-987-29873-0-5

(10) Ruiz, M. A., Correa, E., Cantón, M. A. (2012) Función Ambiental de Parques Urbanos en Zonas Áridas: Clima y Confort Térmico. XIV ENTAC - Encontro Nacional de Tecnologia do Ambiente Construído - Juiz de Fora.

(11) Stocco, S., Cantón, A., Correa, E., (2015) Design of urban green square in dry areas: Thermal performance and comfort. Urban Forestry \& Urban Greening, ${ }^{\circ} 14$ 323-335.

(12) Manat, S., Kazunori, H. (2013) Evaluating the cooling effects of greening for improving the outdoor thermal environment at an institutional campus in the summer. Building and Environment 66158 - 72. http://dx.doi.org/10.1016/j. buildenv.2013.04.012

(13) Song, B., Park, K. (2015).Contribution of Greening and High-Albedo Coatings to Improvements in the Thermal Environment in Complex Urban Areas. Advances in Meteorology, Article ID 792172, 14, http://dx.doi.org/10.1155/2015/792172.

(14) Skelhorn, C., Lindley, S., Levermore, G. (2014) The impact of vegetation types on air and surface temperatures in a temperate city: A fine scale assessment in Manchester, UK Landscape and Urban Planning 121, 129-140.

(15) http://www.geezar.es/ursos-sostenibilidad-a-escala-de-barrio/

(16) Tumini, I., Higueras-García, E. (2012). Alcance y limitaciones de las herramientas de simulación para el estudio del microclima urbano. DYNA Energía y Sostenibilidad. Planificación urbana. Medio urbano. 2: 1-17 DOI: http://dx.doi.org/10.6036/ES6921

(17) Bruse, M. (2011) ENVI-met (Version 3.1 BETA V). http://www.envi-met.com/

(18) Krüger, E., Minella, F., Rasia, F., (2011) Impact of urban geometry on outdoor thermal comfort and air quality from field measurements in Curitiba, Brazil. Building and Environment. 46(3) 621-634. http://dx.doi.org/10.1016/j.buildenv.2010.09.006

(19) Wania, A., Bruse, M., Blond, N., Weber, C. (2012). Analysing the influence of different street vegetation on traffic-induced particle dispersion using microscale simulations. Journal of Environmental Management, 94, 91-101.

(20) Yang, S., Lin, T. (2016) An integrated outdoor spaces design procedure to relieve heat stress in hot and humid regions. Building and Environment, 99, 149-160.

(21) Yuan, O. J., Emura, K., Farnham, C. (2017) Is urban albedo or urban green covering more effective for urban microclimate improvement?: A simulation for Osaka. Sustainable Cities and Society, 32, 78-86

(22) Bruse, M. (1999) Die Auswirkungen kleinskaliger Umweltgestaltung auf das Mikroklima. Entwicklung des prognostischen numerischen Modells ENVI-met zur Simulation der Wind-, Temperatur-, und Feuchtverteilung in städtischen Strukturen. PhD Thesis, Univ. Bochum, Germany.

(23) Ali Toudert, F. (2005). Dependence of outdoor thermal comfort on street design in hot and dry climate. Berichte des Meteorologischen Institutes der Universitat Freiburg. 15 http://www.freidok.uni-freiburg.de/volltexte/2078

(24) González Viveros, P. J. (2011). Evaluación microclimática por cambio de uso de suelo en la Ciudad de México utilizando el modelo ENVI-met (Tesis de Licenciatura). Facultad de Instrumentación Electrónica y de Ciencias Atmosféricas, Universidad de Veracruz, Xalapa-Equez., Veracruz, México.

(25) Samaali, M., Dominique, C., Bruse, M., Olioso, A. \& Occelli, R. (2007). Analysis of a 3D boundary layer model at local scale: Validation on soybean surface radiative measurements. Atmospheric Research, 85(2), 183-198. DOI: 10.1016/j. atmosres.2006.12.005

(26) Chow, W. T., Pope, R. L., Martin, C. A., Brazel, A. J. (2011) Observing and modeling the nocturnal park cool island of an arid city: horizontal and vertical impacts. Theor Appl Climatol;103:197e211.

(27) Middel, A., Häb, K., Brazel, A. J., Chris A. Martin, C. A., Guhathakurta. S. (2014) Impact of urban form and design on mid-afternoon microclimate in Phoenix Local Climate Zones, Landscape and Urban Planning 122 16-28

(28) Song, B., Park, K. (2015) Contribution of Greening and High-Albedo Coatings to Improvements in the Thermal Environment in Complex Urban Areas. Hindawi Publishing Corporation, Advances in Meteorology, Article ID 792172, 14 http:// dx.doi.org/10.1155/2015/792172 
(29) Kottek, M., Grieser, J., Beck, C., Rudolf, B., Rubel, F. (2006). World map of the Köppen-Geiger climate classification updated. Meteorol Z 15(3): 259-263. doi:10.1127/0941-2948/2006/0130

(30) Mendoza Aero Observations SAME. Francisco Gabrielli Airport (2014) Station number: 87418. http://www.wunderground.com/history/airport/SAME/

(31) Pattini, A. E. \& J. M. Monteoliva, (2014) Luminancia/irradiancia en la estación de medición IDMP-CCT CONICET Mendoza. $5^{\circ}$ Congreso Internacional Solar Cities "Energía en las ciudades: innovación frente al cambio climático"

(32) Sosa, M. B., Correa, E. N., Cantón, M. A. (2017) Urban grid forms as a strategy for reducing heat island effects in arid cities. Sustainable Cities and Society 32 547-556

(33) Givoni, B. (1998). Climate considerations in building and urban design. John Wiley \& Sons, Inc. New York, pp 241. 